\title{
A Production Inventory Model for Weibull Deteriorating Items with Two Components of Demand Rate
}

\author{
Sushil Kumar', Uday Singh Rajput ${ }^{2}$ \\ ${ }^{1}$ Department of Mathematics \&Astronomy, University of Lucknow, Lucknow, U.P. (India). \\ ${ }^{2}$ Department of Mathematics \&Astronomy, University of Lucknow, Lucknow, U.P. (India).
}

*Corresponding Author: Sushil Kumar, Department of Mathematics \&Astronomy, University of Lucknow, Lucknow, U.P. (India).

\begin{abstract}
The present paper deals a production inventory model for weibull deteriorating items with constant and exponential demand rate. The products of this model have been considered to have finite life and small amount of decay. The rate of production is constant. The production starts with zero inventory level and stop at a desired maximum inventory level. The purpose of our study is to optimizing the total variable inventory cost. A numerical example is also given to demonstrate the developed model.
\end{abstract}

Keywords: Production Inventory, Weibull Deterioration, Two Component Demand Rate and Constant Production Rate.

\section{INTRODUCTION}

Academicians as well as industrialists have great interest in the development of inventory control and their uses. There are many goods that are either deteriorates or become obsolete with passage of time, such perishable products have different modeling. Perishable inventory forms a small portion of the total inventory. The perishable inventory items can be classified into three categories: (1) deterioration (2) obsolescence (3) no deterioration or no obsolescence. Obsolescence occurs due to the arrival of new and better products in the market.

In the existing literature several inventory models were constructed by several researchers based on the market demand. They have assumed that the demand rate is either constant or an increasing or decreasing function of time or stock dependent. The demand of newly launched products, such as fashionable garments, electronic items, mobiles, motor vehicles etc. increases with time and later it becomes constant.

Rosenberg [1979] presented a lot-size inventory model for deteriorating items with partial backlogging. Billington [1987] developed an economic production quantity model by considering ordering cost as a function of capital expenditure. Urban [1995] presented an inventory model of deteriorating items with stock dependent demand rate by allowing shortages. Abad [1996] proposed an inventory model and studied the optimal pricing and lot sizing conditions of deterioration and partial backlogging. Kishan and Mishra [1996] presented an inventory model with exponential demand rate. They also considered constant deterioration rate and shortages in their inventory model. Teng et al. [1999] proposed a lot-size inventory model for deteriorating items with fluctuating demand and allowing shortages. Skouri and Papachristos [2002] developed a continuous review inventory model for deteriorating items with time dependent demand rate and partial backlogging.

Ouyang and Cheng [2005] presented an inventory model of deteriorating items with exponential declining demand and partial backlogging. Ouyang et al. [2005] proposed an inventory model for deteriorating items with exponential declining demand by allowing shortages. Chund and Wee [2008] discussed the scheduling and replenishment plan for an integrated inventory model of deteriorating items with stock dependent selling rate. Jain et al. [2008] developed an inventory model of deteriorating items with inventory level declining demand rate and allowing shortages. Mishra et al. [2013] proposed an inventory model for deteriorating items with time dependent demand rate and holding cost by allowing shortages. Dash et al. [2014] presented an inventory model of deteriorating 
items with exponential declining demand rate and time varying holding cost. Raj et al. [2015] discussed an inventory model for deteriorating items with exponential demand rate and partial backlogging. Islam [2015, 2016] developed two inventory models. One of them is a production inventory model of deteriorating items with constant demand rate and three types of production rate and other is an inventory model with exponential demand rate and constant production rate for the products having finite shelf-life. Islam et al. [2015] presented a production inventory model with constant production rate and different components of demand. They also considered the product's shelf -life is finite in their inventory model. Ukil et al. [2015] discussed a production inventory model with constant production rate and time dependent power demand by considering the finite shelf -life of products. Lakshmidevi and Maragatham [2015] developed an inventory model with three rates of production and time dependent demand rate and deterioration rate. Ukil and Uddin [2016] proposed a production inventory model with constant production rate and linear trend in demand.

In the present paper, we have developed a production inventory model for weibull deteriorating items with constant and exponential demand rate.

\section{ASSUMPTIONS AND NOTATIONS}

We consider the following assumptions and notations corresponding to the developed model.

1. The demand rate is $R(t)= \begin{cases}a, & 0 \leq t \leq T_{1} \\ a e^{b t}, & T_{1} \leq t \leq T\end{cases}$ where $a$ and $b$ are constants.

2. The deterioration rate is $\boldsymbol{\theta}(t)=\boldsymbol{\alpha} \boldsymbol{\beta} t^{\beta-1}$, where $\alpha$ and $\beta$ are parameters.

3. The production rate is $P(t)=\lambda$, where $\lambda$ is a constant.

4. $o_{C}$ is the ordering cost per order.

5. $h_{C}$ is the holding cost per unit per cycle.

6. $d_{C}$ is the deterioration cost per unit per cycle.

7. $\mathrm{T}$ is the cycle length.

8. $T_{1}$ is the time at which production level reaches maximum.

9. The replenishment rate is finite.

10. The lead time is zero.

11. $T C\left(T_{1}, T\right)$ is the total cost per cycle.

12. $I(t)$ is the inventory level at any time $\mathrm{t}$.

\section{Mathematical Formulation}

Suppose the production starts at time $T=0$ with zero inventory level and becomes maximum inventory level $\mathrm{Q}$ at time $T=T_{1}$. The instantaneous inventory level at any time $\mathrm{t}$ in $[0, T]$ is given by the following differential equations

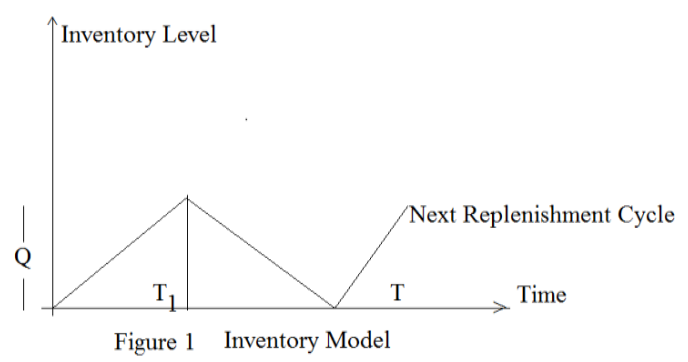


$\frac{d I}{d t}+\theta(t) I=P(t)-R(t)$, putting the values of $P(t)$ and $R(t)$ in this equation, we obtain

$\frac{d I}{d t}+\alpha \beta t^{\beta-1} I=(\lambda-a), \quad 0 \leq t \leq T_{1}$

With initial boundary condition

$$
\begin{aligned}
& I(0)=0 \\
& \frac{d I}{d t}+\alpha \beta t^{\beta-1} I=a e^{b t}, \quad T_{1} \leq t \leq T
\end{aligned}
$$

With initial boundary condition

$$
I\left(T_{1}\right)=Q
$$

The solutions of equations (1) and (2) are given by the equations (3) and (4) respectively.

$$
\begin{aligned}
I(t)= & (\lambda-a) t-\alpha(\lambda-a) t^{\beta+1}, \quad 0 \leq t \leq T_{1} \\
I(t)= & a\left[T_{1}+\left(\frac{b}{2}\right) T_{1}^{2}+\left(\frac{\alpha}{\beta+1}\right) T_{1}^{\beta+1}+\left(\frac{\alpha b}{\beta+2}\right) T_{1}^{\beta+2}-\alpha T_{1} t^{\beta}-\left(\frac{\alpha b}{2}\right) T_{1}^{2} t^{\beta}\right]+Q\left(1+\alpha T_{1}^{\beta}-\alpha t^{\beta}\right) \\
& -a\left[t+\left(\frac{b}{2}\right) t^{2}+\left(\frac{\alpha}{\beta+1}\right) t^{\beta+1}+\left(\frac{\alpha b}{\beta+2}\right) t^{\beta+2}-\alpha t^{\beta+1}-\left(\frac{\alpha b}{2}\right) t^{\beta+2}\right]
\end{aligned}
$$

The maximum inventory level $\mathrm{Q}$ is obtained by putting $t=T_{1}$ in equation (3). We have

$$
Q=(\lambda-a) T_{1}-\alpha(\lambda-a) T_{1}^{\beta+1},
$$

Putting the value of $\mathrm{Q}$ in equation (4), the equation (4) becomes

$$
\begin{aligned}
& I=-a\left[t+\left(\frac{b}{2}\right) t^{2}-\left(\frac{\alpha \beta}{\beta+1}\right) t^{\beta+1}-\left(\frac{b \alpha \beta}{2(\beta+2)}\right) t^{\beta+2}\right]+\lambda T_{1}+\left(\frac{a b}{2}\right) T_{1}^{2}+\left(\frac{a \alpha(2 \beta+3)}{\beta+1}\right) T_{1}^{\beta+1} \\
& -2 \alpha \lambda T_{1}^{\beta+1}+\left(\frac{a b \alpha}{\beta+2}\right) T_{1}^{\beta+2}-\left(\frac{a b \boldsymbol{\alpha}}{2}\right) T_{1}^{2} t^{\beta}-\alpha \lambda T_{1} t^{\beta}, \quad T_{1} \leq t \leq T
\end{aligned}
$$

The ordering cost per cycle is

$$
O_{C}=o_{C}
$$

The holding cost per cycle is

$$
H_{C}=h_{C}\left[\int_{0}^{T_{1}} I(t) d t+\int_{T_{1}}^{T} I(t) d t\right]
$$

Or

$$
\begin{aligned}
H_{C}= & h_{C}\left[-\left(\frac{\lambda}{2}\right) T_{1}^{2}-\left(\frac{a}{2}\right) T^{2}+\lambda T T_{1}-\left(\frac{a b}{3}\right) T_{1}^{3}-\left(\frac{a b}{6}\right) T^{3}+\left(\frac{a b}{2}\right) T T_{1}^{2}-\left(\frac{\alpha \lambda}{\beta+1}\right) T_{1} T^{\beta+1}\right. \\
& +\left\{\frac{a \boldsymbol{\alpha}(2 \boldsymbol{\beta}+3)-2 \alpha \lambda(\boldsymbol{\beta}+1)}{\boldsymbol{\beta}+1}\right\} T T_{1}^{\beta+1}-\left\{\frac{\boldsymbol{\alpha}(a-\lambda)(16 \boldsymbol{\beta}+5)}{7 \boldsymbol{\beta}+2}\right\} T_{1}^{\beta+2}+\left(\frac{a \boldsymbol{\alpha} \boldsymbol{\beta}}{3 \boldsymbol{\beta}+2}\right) T^{\beta+2} \\
& \left.-\left(\frac{a b \boldsymbol{\alpha} \boldsymbol{\beta}}{7 \boldsymbol{\beta}+3}\right) T_{1}^{\beta+3}+\left(\frac{a b \boldsymbol{\alpha} \boldsymbol{\beta}}{2(5 \boldsymbol{\beta}+6)}\right) T^{\beta+3}+\left(\frac{a b \boldsymbol{\alpha}}{\boldsymbol{\beta}+2}\right) T T_{1}^{\beta+2}-\left(\frac{a b \boldsymbol{\alpha}}{2(\boldsymbol{\beta}+1)}\right) T_{1}^{2} T^{\beta+1}\right]
\end{aligned}
$$

The deterioration cost per cycle is

$$
D_{C}=d_{C}\left[\int_{0}^{T_{1}} \theta(t) I(t) d t+\int_{T_{1}}^{T} \theta(t) I(t) d t\right]
$$

Or 


$$
\begin{aligned}
D_{C}= & d_{C}\left[-\left(\frac{\alpha \lambda}{\beta+1}\right) T_{1}^{\beta+1}-\left(\frac{a \boldsymbol{\alpha} \beta}{\beta+1}\right) T^{\beta+1}-\left(\frac{a b \boldsymbol{\alpha}}{\boldsymbol{\beta}+2}\right) T_{1}^{\beta+2}-\left(\frac{a b \boldsymbol{\alpha} \beta}{2(\boldsymbol{\beta}+2)}\right) T^{\beta+2}+\alpha \pi_{1} T^{\beta}\right. \\
& \left.+\left(\frac{a b \boldsymbol{\alpha}}{2}\right) T_{1}^{2} T^{\beta}\right]
\end{aligned}
$$

The total cost per cycle is

$$
T C\left(T_{1}, T\right)=\left(\frac{1}{T}\right)\left[O_{C}+H_{C}+D_{C}\right]
$$

Putting the values of $O_{C}, H_{C}$ and $D_{C}$ in equation (9), we have

$$
\begin{aligned}
T C\left(T_{1}, T\right) & =\left(\frac{1}{T}\right)\left[O_{C}-\left(\frac{\lambda h_{C}}{2}\right) T_{1}^{2}-\left(\frac{a h_{C}}{2}\right) T^{2}+\lambda h_{C} T T_{1}-\left(\frac{a b h_{C}}{3}\right) T_{1}^{3}-\left(\frac{a b h_{C}}{6}\right) T^{3}+\left(\frac{a b h_{C}}{2}\right) T T_{1}^{2}\right. \\
& -\left(\frac{\alpha \lambda d_{C}}{\boldsymbol{\beta}+1}\right) T_{1}^{\beta+1}-\left(\frac{a \boldsymbol{\alpha} \beta d_{C}}{\beta+1}\right) T^{\beta+1}-\left\{\frac{\boldsymbol{\alpha}(a-\lambda) h_{C}(37 \boldsymbol{\beta}+10)+a b \boldsymbol{\alpha} d_{C}(7 \boldsymbol{\beta}+2)}{4(\boldsymbol{\beta}+1)}\right\} T_{1}^{\beta+2} \\
& +\left\{\frac{a \boldsymbol{\alpha} \boldsymbol{\beta}\left(4 h_{C}+2 b d_{C}\right)}{8(2 \boldsymbol{\beta}+1)}\right\} T^{\beta+2}-\boldsymbol{\alpha} \lambda d_{C} T_{1} T^{\beta}-\left(\frac{a b \boldsymbol{\alpha d}}{2}\right) T_{1}^{2} T^{\beta}-\left(\frac{\boldsymbol{\alpha} \lambda h_{C}}{\boldsymbol{\beta}+1}\right) T_{1} T^{\beta+1} \\
& +\left\{\frac{a \boldsymbol{\alpha}(2 \boldsymbol{\beta}+3)-2 \boldsymbol{\alpha} \lambda(\boldsymbol{\beta}+1)}{\boldsymbol{\beta}+1}\right\} h_{C} T T_{1}^{\beta+1}-\left(\frac{a b \boldsymbol{\alpha} \boldsymbol{\beta} h_{C}}{7 \boldsymbol{\beta}+3}\right) T_{1}^{\beta+3}+\left(\frac{a b \boldsymbol{\alpha} \boldsymbol{\beta} h_{C}}{2(5 \boldsymbol{\beta}+6)}\right) T^{\beta+3} \\
& \left.+\left(\frac{a b \boldsymbol{\alpha} h_{C}}{\boldsymbol{\beta}+2}\right) T T_{1}^{\beta+2}-\left(\frac{a b \boldsymbol{\alpha} h_{C}}{2(\boldsymbol{\beta}+1)}\right) T_{1}^{2} T^{\beta+1}\right]
\end{aligned}
$$

The necessary conditions for $T C\left(T_{1}, T\right)$ to be minimum are

$$
\frac{\partial T C\left(T_{1}, T\right)}{\partial T_{1}}=0, \quad \frac{\partial T C\left(T_{1}, T\right)}{\partial T}=0
$$

Solving these equations, we find the optimum values of $T_{1}$ and $T$ for which the total cost is minimum. The sufficient conditions for $T C\left(T_{1}, T\right)$ are

$$
\left(\frac{\partial^{2} T C\left(T_{1}, T\right)}{\partial T_{1}^{2}}\right)\left(\frac{\partial^{2} T C\left(T_{1}, T\right)}{\partial T^{2}}\right)-\left(\frac{\partial^{2} T C\left(T_{1}, T\right)}{\partial T_{1}^{2}}\right)^{2}>0 \text {, and }\left(\frac{\partial^{2} T C\left(T_{1}, T\right)}{\partial T_{1}^{2}}\right)>0
$$

Differentiating equation (10), we obtain

$$
\begin{aligned}
\frac{\partial T C\left(T_{1}, T\right)}{\partial T_{1}} & =\left(\frac{1}{T}\right)\left[-\lambda h_{C} T_{1}+\lambda h_{C} T-a b h_{C} T_{1}^{2}+a b h_{C} T T_{1}-\alpha \lambda d_{C} T_{1}^{\beta}-\alpha \lambda d_{C} T^{\beta}-a b \alpha \lambda d_{C} T_{1} T^{\beta}\right. \\
& -\left\{\frac{\alpha(a-\lambda) h_{C}(21 \beta+5)+a b \alpha d_{C}(4 \beta+1)}{4 \beta+1}\right\} T_{1}^{\beta+1}+\{a \alpha(2 \beta+3)-2 \alpha \lambda(\beta+1)\} h_{C} T T_{1}^{\beta} \\
& \left.-\left(\frac{\alpha h_{C}}{\beta+1}\right) T^{\beta+1}-\left(\frac{3 a b \boldsymbol{\alpha} \beta h_{C}}{7 \beta+3}\right) T_{1}^{\beta+2}+a b \boldsymbol{\alpha} h_{C} T T_{1}^{\beta+1}-\left(\frac{a b \boldsymbol{\alpha} h_{C}}{\beta+1}\right) T_{1} T^{\beta+1}\right]
\end{aligned}
$$

$$
\begin{gathered}
\frac{\partial T C\left(T_{1}, T\right)}{\partial T}=\left(\frac{1}{T}\right)\left[-a h_{C} T+\lambda h_{C} T_{1}-\left(\frac{a b h_{C}}{2}\right) T^{2}+\left(\frac{a b h_{C}}{2}\right) T_{1}^{2}-a \alpha \beta d_{C} T^{\beta}-\alpha \beta \lambda d_{C} T_{1} T^{\beta-1}\right. \\
+\left\{\frac{a \alpha \beta\left(2 h_{C}+b d_{C}\right)}{2(2 \beta+1)}\right\} T^{\beta+1}-\left(\frac{a b \alpha \beta d_{C}}{2}\right) T_{1}^{2} T^{\beta-1}+\left\{\frac{a \alpha(2 \beta+3)-2 \alpha \lambda(\beta+1)}{(\beta+1)}\right\} h_{C} T_{1}^{\beta+1}
\end{gathered}
$$




$$
\begin{aligned}
& \left.-\alpha \lambda h_{C} T_{1} T^{\beta}+\left(\frac{3 a b \boldsymbol{\alpha} \beta h_{C}}{2(5 \boldsymbol{\beta}+6)}\right) T^{\beta+2}+\left(\frac{a b \boldsymbol{\alpha} h_{C}}{\boldsymbol{\beta}+2}\right) T_{1}^{\beta+2}-\left(\frac{a b \boldsymbol{\alpha} h_{C}}{2}\right) T_{1}^{2} T^{\beta}\right]-\left(\frac{1}{T^{2}}\right)\left[O_{C}\right. \\
& -\left(\frac{\lambda h_{C}}{2}\right) T_{1}^{2}-\left(\frac{a h_{C}}{2}\right) T^{2}+\lambda h_{C} T T_{1}-\left(\frac{a b h_{C}}{3}\right) T_{1}^{3}-\left(\frac{a b h_{C}}{6}\right) T^{3}+\left(\frac{a b h_{C}}{2}\right) T T_{1}^{2}-\left(\frac{\alpha \lambda d_{C}}{\beta+1}\right) T_{1}^{\beta+1} \\
& -\left(\frac{a \boldsymbol{\alpha} \beta d_{C}}{\boldsymbol{\beta}+1}\right) T^{\beta+1}-\left\{\frac{\boldsymbol{\alpha}(a-\lambda) h_{C}(37 \boldsymbol{\beta}+10)+a b \boldsymbol{\alpha} d_{C}(7 \boldsymbol{\beta}+2)}{4(4 \boldsymbol{\beta}+1)}\right\} T_{1}^{\beta+2}-\alpha \lambda d_{C} T_{1} T^{\beta} \\
& +\left\{\frac{a \boldsymbol{\alpha}\left(2 h_{C}+b d_{C}\right)}{4(2 \boldsymbol{\beta}+1)}\right\} T^{\beta+2}-\left(\frac{a b \boldsymbol{\alpha} d_{C}}{2}\right) T_{1}^{2} T^{\beta}+\left\{\frac{a \boldsymbol{\alpha}(2 \boldsymbol{\beta}+3)-2 \alpha \lambda(\boldsymbol{\beta}+1)}{\beta+1}\right\} h_{C} T T_{1}^{\beta+1} \\
& -\left(\frac{\boldsymbol{\alpha} \lambda h_{C}}{\boldsymbol{\beta}+1}\right) T_{1} T^{\beta+1}-\left(\frac{a b \boldsymbol{\beta} h_{C}}{7 \boldsymbol{\beta}+3}\right) T_{1}^{\beta+3}+\left(\frac{a b \boldsymbol{\alpha} h_{C}}{2(5 \boldsymbol{\beta}+6)}\right) T^{\beta+3}+\left(\frac{a b \boldsymbol{\alpha} h_{C}}{\beta+2}\right) T T_{1}^{\beta+2} \\
& \left.-\left(\frac{a b \boldsymbol{\alpha} h_{C}}{2(\boldsymbol{\beta}+1)}\right) T_{1}^{2} T^{\beta+1}\right]
\end{aligned}
$$

$\frac{\partial^{2} T C\left(T_{1}, T\right)}{\partial T_{1}^{2}}=\left(\frac{1}{T}\right)\left[-\lambda h_{C}-2 a b h_{C} T_{1}+a b h_{C} T-\alpha \beta \lambda d_{C} T_{1}^{\beta-1}-a b \alpha d_{C} T^{\beta}\right.$

$$
\begin{aligned}
& -(\boldsymbol{\beta}+1)\left\{\frac{\alpha(a-\lambda) h_{C}(21 \boldsymbol{\beta}+5)+a b \boldsymbol{\alpha} d_{C}(4 \boldsymbol{\beta}+1)}{4 \boldsymbol{\beta}+1}\right\} T_{1}^{\beta}+\alpha \beta\{a(2 \boldsymbol{\beta}+3)-2 \lambda(\boldsymbol{\beta}+1)\} h_{C} T T_{1}^{\beta-1} \\
& \left.-\left(\frac{3 a b \boldsymbol{\alpha} \beta(\boldsymbol{\beta}+2) h_{C}}{7 \boldsymbol{\beta}+3}\right) T_{1}^{\beta+1}-\left(\frac{a b \boldsymbol{\alpha} h_{C}}{\boldsymbol{\beta}+1}\right) T^{\beta+1}+a b \boldsymbol{\alpha}(\boldsymbol{\beta}+1) h_{C} T T_{1}^{\beta}\right]
\end{aligned}
$$

$\frac{\partial^{2} T C\left(T_{1}, T\right)}{\partial T_{1} \partial T}=\left(\frac{1}{T}\right)\left[\lambda h_{C}+a b h_{C} T_{1}-\alpha \beta \lambda d_{C} T^{\beta-1}-a b \alpha \beta d_{C} T_{1} T^{\beta-1}+\{a \alpha(2 \beta+3)-2 \alpha \lambda(\beta+1)\} h_{C} T_{1}^{\beta}\right.$

$\left.-\alpha \lambda h_{C} T^{\beta}+a b \alpha h_{C} T_{1}^{\beta+1}-a b \alpha h_{C} T_{1} T^{\beta}\right]+\left(\frac{2}{T^{3}}\right)\left[-\lambda h_{C} T_{1}+\lambda h_{C} T-a b h_{C} T_{1}^{2}\right.$

$+a b h_{C} T T_{1}-\alpha \lambda d_{C} T_{1}^{\beta}-(\beta+2)\left\{\frac{\alpha(a-\lambda)(37 \beta+10) h_{C}+a b \alpha d_{C}(7 \beta+2)}{4(4 \beta+1)}\right\} T_{1}^{\beta+1}$

$-\alpha \lambda d_{C} T^{\beta}-a b \alpha d_{C} T_{1} T^{\beta}+\{a \alpha(2 \beta+3)-2 \alpha \lambda(\beta+1)\} h_{C} T T_{1}^{\beta}-\left(\frac{\alpha \lambda h_{C}}{\beta+1}\right) T^{\beta+1}$

$\left.-\left(\frac{a b \alpha \beta(\beta+3) h_{C}}{7 \beta+3}\right) T_{1}^{\beta+2}+a b \alpha h_{C} T T_{1}^{\beta+1}-\left(\frac{a b \alpha h_{C}}{\beta+1}\right) T_{1} T^{\beta+1}\right]$

$$
\begin{aligned}
& \frac{\partial^{2} T C\left(T_{1}, T\right)}{\partial T^{2}}=\left(\frac{1}{T}\right)\left[-a h_{C}-a b h_{C} T-a \alpha \beta^{2} d_{C} T^{\beta-1}+\left(\frac{a \boldsymbol{\alpha}(\boldsymbol{\beta}+1)\left(2 h_{C}+b d_{C}\right)}{2(2 \boldsymbol{\beta}+1)}\right) T^{\beta}\right. \\
& -\alpha \beta \lambda(\beta-1) d_{C} T_{1} T^{\beta-2}-\left(\frac{a b \boldsymbol{\alpha}(\boldsymbol{\beta}-1) d_{C}}{2}\right) T_{1}^{2} T^{\beta-2}-\alpha \beta \lambda h_{C} T_{1} T^{\beta-1}+\left(\frac{3 a b \boldsymbol{\alpha}(\boldsymbol{\beta}+2) h_{C}}{2(5 \beta+6)}\right) T^{\beta+1}
\end{aligned}
$$$$
\left.-\left(\frac{a b \alpha \beta h_{C}}{2}\right) T_{1}^{2} T^{\beta-1}\right]-\left(\frac{1}{T^{2}}\right)\left[-2 a h_{C} T+2 \lambda h_{C} T_{1}-a b h_{C} T^{2}+a b h_{C} T_{1}^{2}-2 a \alpha \beta d_{C} T^{\beta}\right.
$$$$
+\left(\frac{a \alpha \beta(\beta+3)\left(2 h_{C}+b d_{C}\right)}{4(2 \beta+1)}\right) T^{\beta+1}-2 \alpha \beta \lambda d_{C} T_{1} T^{\beta-1}-\left(\frac{a b \alpha \beta d_{C}}{2}\right) T_{1}^{2} T^{\beta-1}
$$ 


$$
\begin{aligned}
& +2\left\{\frac{a \alpha(2 \beta+3)-2 \alpha \lambda(\beta+1)}{\beta+1}\right\} h_{C} T_{1}^{\beta+1}-2 \alpha \lambda h_{C} T_{1} T^{\beta}+\left(\frac{a b \alpha \beta(\beta+6) h_{C}}{2(5 \beta+6)}\right) T^{\beta+2}+\left(\frac{2 a b \alpha h_{C}}{\beta+2}\right) T_{1}^{\beta+2} \\
& \left.-a b \alpha h_{C} T_{1}^{2} T^{\beta}\right]+\left(\frac{2}{T^{3}}\right)\left[o_{C}-\left(\frac{\lambda h_{C}}{2}\right) T_{1}^{2}-\left(\frac{a h_{C}}{2}\right) T^{2}+\lambda h_{C} T T_{1}-\left(\frac{a b h_{C}}{3}\right) T_{1}^{3}-\left(\frac{a b h_{C}}{6}\right) T^{3}\right. \\
& +\left(\frac{a b h_{C}}{2}\right) T T_{1}^{2}-\left(\frac{\alpha \lambda d_{C}}{\beta+1}\right) T_{1}^{\beta+1}-\left(\frac{a \alpha \beta \lambda d_{C}}{\beta+1}\right) T^{\beta+1}-\left\{\frac{\alpha(a-\lambda) h_{C}(37 \boldsymbol{\beta}+10)+a b \alpha d_{C}(7 \boldsymbol{\beta}+2)}{4(4 \boldsymbol{\beta}+1)}\right\} T_{1}^{\beta+2} \\
& +\left(\frac{a \alpha \beta\left(2 h_{C}+b d_{C}\right)}{4(2 \beta+1)}\right) T^{\beta+2}-\alpha \lambda d_{C} T_{1} T^{\beta}-\left(\frac{a b \alpha d_{C}}{2}\right) T_{1}^{2} T^{\beta}-\left(\frac{\alpha \lambda d_{C}}{\beta+1}\right) T_{1} T^{\beta+1} \\
& +\left\{\frac{a \boldsymbol{\alpha}(2 \boldsymbol{\beta}+3)-2 \alpha \lambda(\boldsymbol{\beta}+1)}{\boldsymbol{\beta}+1}\right\} h_{C} T T_{1}^{\beta+1}-\left(\frac{a b \boldsymbol{\alpha} \beta \lambda_{C}}{7 \boldsymbol{\beta}+3}\right) T_{1}^{\beta+3}+\left(\frac{a b \boldsymbol{\alpha} \beta h_{C}}{2(5 \boldsymbol{\beta}+6)}\right) T^{\beta+3} \\
& \left.+\left(\frac{a b \boldsymbol{\alpha} h_{C}}{\boldsymbol{\beta}+2}\right) T T_{1}^{\beta+2}-\left(\frac{a b \boldsymbol{\alpha} \lambda h_{C}}{2(\boldsymbol{\beta}+1)}\right) T_{1}^{2} T^{\beta+1}\right]
\end{aligned}
$$

\section{NUMERICAL EXAMPLE}

Let we consider the following data for parameters of the model in appropriate units $a=10, \quad b=0.3, \quad \alpha=3, \quad \beta=1, \quad o_{C}=15, \quad h_{C}=0.4, \quad d_{C}=0.4, \quad \lambda=500$

\section{TABLES \& FigURES}

Table 1. variation of total inventory cost with respect to $\alpha$

\begin{tabular}{|l|l|l|l|}
\hline$\alpha$ & $T_{1}$ & $T$ & $T C\left(T_{1}, T\right)$ \\
\hline 3 & 13144.30 & 20814.60 & $4.0986 \times 10^{11}$ \\
6 & 13169.10 & 20854.80 & $7.7719 \times 10^{11}$ \\
9 & 13177.30 & 20868.30 & $1.1941 \times 10^{12}$ \\
12 & 13169.90 & 20854.60 & $1.5875 \times 10^{12}$ \\
15 & 13171.50 & 20858.70 & $1.9861 \times 10^{12}$ \\
\hline
\end{tabular}

From this table, we see that as we increase the parameter $\alpha$, then the values of $T_{1}, T$ and $\operatorname{TC}\left(T_{1}, T\right)$ are increased.

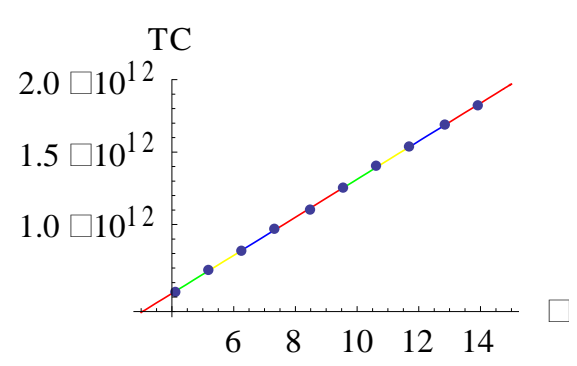

Figure 2. variation in $T C\left(T_{1}, T\right)$ with $\alpha$,

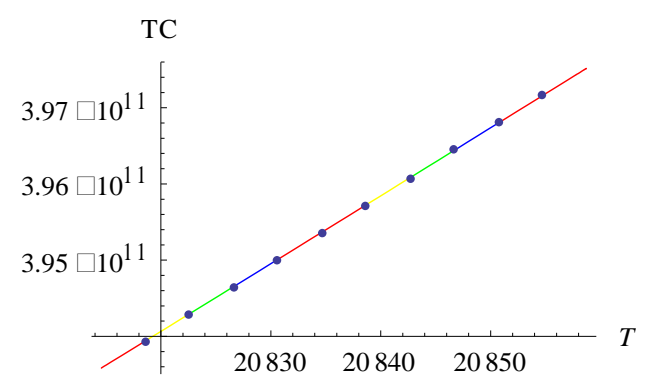

Figure 3. variation in $T C\left(T_{1}, T\right)$ with $T$

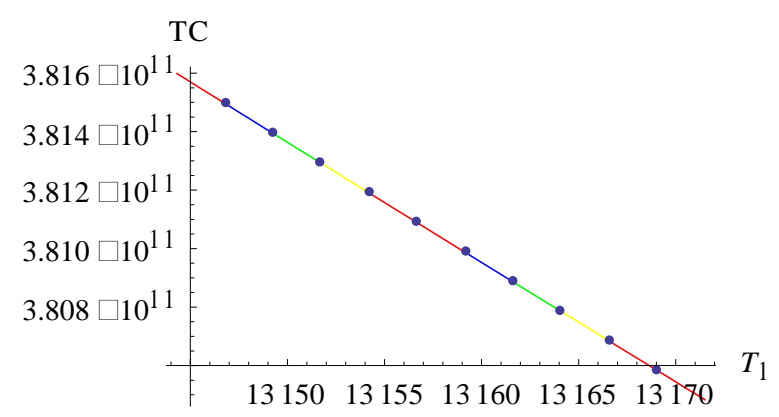

Figure 4. variation in $T C\left(T_{1}, T\right)$ with $T_{1}$ 
Table 2. variation in total inventory cost with respect to a

\begin{tabular}{|l|l|l|l|}
\hline$a$ & $T_{1}$ & $T$ & $T C\left(T_{1}, T\right)$ \\
\hline 10 & 13144.30 & 20814.60 & $4.0986 \times 10^{11}$ \\
20 & 6466.42 & 10240.60 & $9.4303 \times 10^{10}$ \\
30 & 4232.15 & 6702.34 & $3.9498 \times 10^{10}$ \\
40 & 3115.02 & 4933.23 & $2.1079 \times 10^{10}$ \\
50 & 2444.75 & 3871.77 & $1.2718 \times 10^{10}$ \\
\hline
\end{tabular}

From this table, we see that as we increase the parameter $a$, the values of $T_{1}, T$ and $T C\left(T_{1}, T\right)$ are decreased.
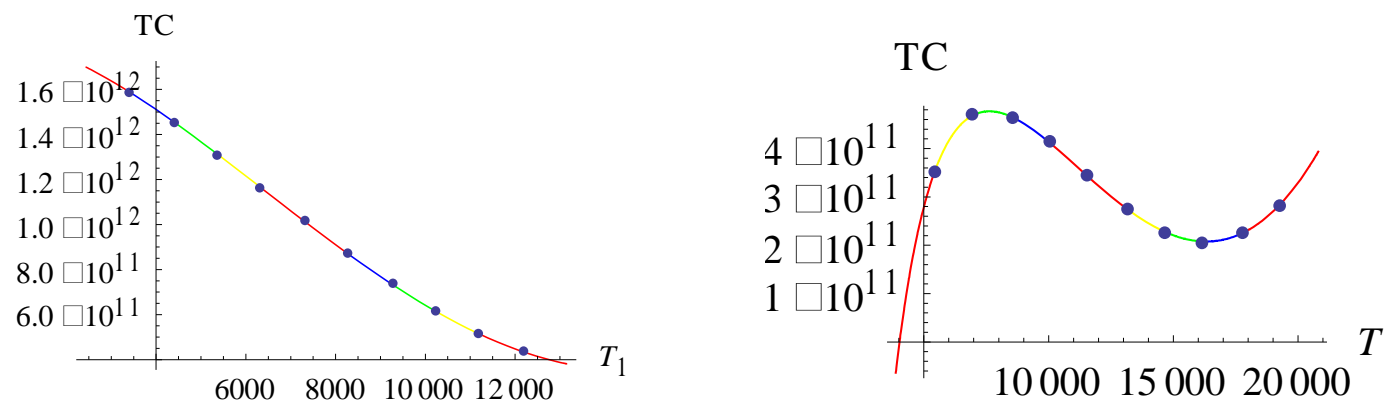

Figure 5. variation in total cost with $T_{1}$,

Figure 6. variation in total cost with $T$

Table 3. variation in total inventory cost with respect to $b$

\begin{tabular}{|l|l|l|l|}
\hline$b$ & $T_{1}$ & $T$ & $T C\left(T_{1}, T\right)$ \\
\hline 0.3 & 13144.30 & 20814.60 & $4.0986 \times 10^{11}$ \\
0.6 & 6594.29 & 10442.00 & $9.9483 \times 10^{11}$ \\
0.9 & 4402.65 & 6970.84 & $4.3301 \times 10^{10}$ \\
1.2 & 3306.82 & 5235.29 & $2.4915 \times 10^{10}$ \\
1.5 & 2649.33 & 4193.95 & $1.6192 \times 10^{10}$ \\
\hline
\end{tabular}

From this table, we see that as we increase the parameter $b$, then the values of $T_{1}, T$ and $T C\left(T_{1}, T\right)$ are decreased.

TC
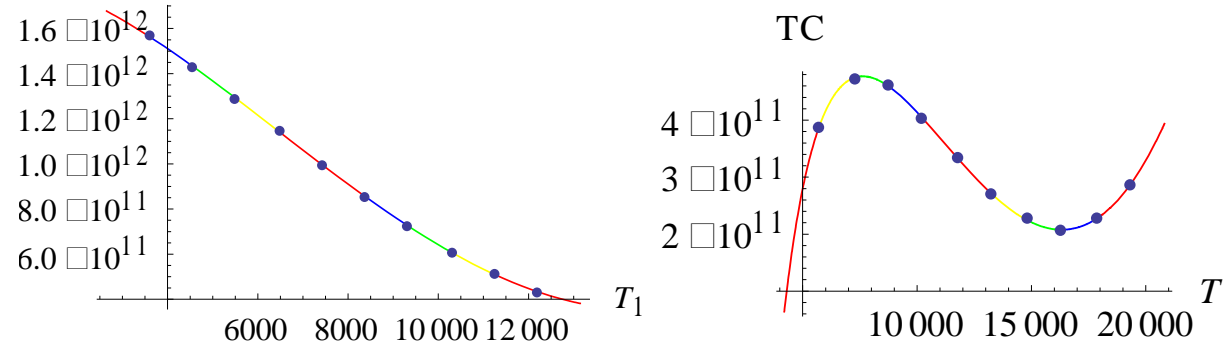

Figure 7. variation in total cost with $T_{1}$,

Figure 8. variation in total cost with $T$

These are the numerical values of second order derivatives

$$
\frac{\partial^{2} T C\left(T_{1}, T\right)}{\partial T_{1}^{2}}=46125.30, \quad \frac{\partial^{2} T C\left(T_{1}, T\right)}{\partial T_{1} \partial T}=-24397.60, \quad \frac{\partial^{2} T C\left(T_{1}, T\right)}{\partial T^{2}}=9.3129 \times 10^{6}
$$

\section{CONClusion}

In this paper, we have developed a production inventory model for weibull deteriorating items with two components of demand rate. We see that the parameters $\alpha$ is more sensitive than the parameters $a$ 
and $b$. This is the reason that the products having finite life to be sold as soon as possible in the market. Therefore, it is very practical and suitable situation for the products of our daily lives and the firm/retailer wants to increase their demand.

\section{REFERENCES}

[1] Sivazlin B. D. and Stenfel L. E., Analysis of Systems in Operations Research, 1975, 203-230.

[2] Rosenberg D., An Analysis of a Lot-Size Inventory Model for Deteriorating Items with Partial Backlogging, Naval Research Logistics Quarterly, 26(2), 349-353, (1979).

[3] Billington P. L., An Economic Production Model with Ordering Cost as a Function of Capital Expenditure, Decision Series, 18, 25-42, (1987).

[4] Urban T. L., An Inventory Model with Stock Dependent Demand Rate by Allowing Shortage, Journal of Product Economic, 40, 21-28, (1995).

[5] Abad P. L., An Optimal Pricing and Lot Sizing Inventory Model of Deteriorating Items by Allowing Shortages, Management Science, 42(8), 1093-1104, (1996).

[6] Kishan H. and Mishra P. N., An Inventory Model for Deteriorating Items with Constant Deterioration Rate and Exponential Demand Rate by Allowing Shortages, Allahabad Mathematical Society, 275- 279, (1996).

[7] Teng J. T., Chern M. S. and Yang H. L., A Deterministic Lot-Size Inventory Model for Deteriorating Items with Fluctuating Demand, Operation Research Letters,24, 65- 72, (1999).

[8] Skouri K. and Papachristos S., A Continuous Review Inventory Model for Deteriorating Items with Time Varying Demand and Partial Backlogging, Applied Mathematical Modeling,26, 603- 617, (2002).

[9] Ouyang L. Y., Wu K. S. and Cheng M. C., An Inventory Model of Deteriorating with Exponential Decreasing Demand and Allowing Shortages, Yugoslav Journal of Operation Research,15(2), 277- 288, (2005).

[10] Chung C. J. and Wee H. M., A Scheduling and Replenishment Plan for an Inventory Model of Deteriorating Items with Stock Dependent Selling Rate, International Journal of Advanced Manufacturing Technology,35(7-8), 665- 679, (2008).

[11] Jain S., Kumar M. and Advan P., An Inventory Model for Deteriorating Items with Partial Backlogging and Stock Dependent Decreasing Demand Rate, International Journal Operations Research,5(3), 154-159, (2008).

[12] Mishra V. K., Singh L. S. and Kumar R., An Inventory Model for Deteriorating Items with Time Dependent Demand and Time Varying Holding Cost by Allowing Shortages, International Journal of Industrial Engineering,9(4), 1-4, (2013).

[13] Dash B. P., Sing T. and Pattnayak H., An Inventory Model for Deteriorating Items with Exponential Declining Demand and Time Varying Holding Cost, American Journal Operations Research, 4,1-7, (2014).

[14] Lakshmidevi P. K. and Maragatham M., An Inventory Model with Three Rates of Production and Time Dependent Deterioration Rate and Quadratic Demand Rate, International Journal of Fuzzy Mathematical Archive,6(1), 99-103, (2015).

[15] Raj R., Kaliraman N. K., Chandra S., and Chaudhury H., An Inventory Model for Deteriorating Items with Exponential Demand Rate and Allowing Shortages, International Journal of Mathematical Trend and Technology,22(1), 9-16, (2015).

[16] Ekramol Islam M., A Production Inventory Model with Three Production Rates and Constant Demand Rate, Bangladesh Islamic University Journal,1(1), 14-20, (2015).

[17] Islam M. E., Ukil S. I. and Uddin M. S., A Production Inventory Model with Constant Production Rate and Different Classes of Demand for the Products having Finite Shelf-life, Proceedings of International Conference on Mechanical, Industrial and Material Engineering (ICMIME)-2015, RUET, Rajshashi, Bangladesh,11-13, (2015).

[18] Ukil S. I., Islam M. E. and Uddin M. S., A Production Inventory Model with Constant Production Rate and Power Demand for the Products having Finite Shelf-Life, Journal of Service Science and Management,8(6), 874- 885, (2015).

[19] Ukil S. L. and Uddin M. S., A Production Inventory Model with Stock Dependent Linear Trend in Demand and Constant Production Rate, American Journal of Operations Research,6, 61-70, (2016). 
Citation: S. Kumar, U. S. Rajput, " A Production Inventory Model for Weibull Deteriorating Items with Two Components of Demand Rate ", International Journal of Scientific and Innovative Mathematical Research, vol. 6, no. 3, p. 29-37, 2018., http://dx.doi.org/10.20431/2347-3142.0603004

Copyright: (c) 2018 Authors. This is an open-access article distributed under the terms of the Creative Commons Attribution License, which permits unrestricted use, distribution, and reproduction in any medium, provided the original author and source are credited. 